\title{
KLEJ: Comprehensive Benchmark for Polish Language Understanding
}

\author{
Piotr Rybak ${ }^{1}$, Robert Mroczkowski ${ }^{1}$, Janusz Tracz $^{1}$, and Ireneusz Gawlik ${ }^{1,2}$ \\ ${ }^{1}$ ML Research at Allegro.pl, \\ ul. Grunwaldzka 182, 60-166 Poznań, Poland \\ \{firstname.lastname\} @allegro.pl \\ ${ }^{2}$ AGH University of Science and Technology \\ Faculty of Computer Science, Electronics and Telecommunications, \\ Department of Computer Science \\ al. A. Mickiewicza 30, 30-059 Kraków, Poland
}

\begin{abstract}
In recent years, a series of Transformer-based models unlocked major improvements in general natural language understanding (NLU) tasks. Such a fast pace of research would not be possible without general NLU benchmarks, which allow for a fair comparison of the proposed methods. However, such benchmarks are available only for a handful of languages. To alleviate this issue, we introduce a comprehensive multi-task benchmark for the Polish language understanding, accompanied by an online leaderboard. It consists of a diverse set of tasks, adopted from existing datasets for named entity recognition, question-answering, textual entailment, and others. We also introduce a new sentiment analysis task for the e-commerce domain, named Allegro Reviews (AR). To ensure a common evaluation scheme and promote models that generalize to different NLU tasks, the benchmark includes datasets from varying domains and applications. Additionally, we release HerBERT, a Transformer-based model trained specifically for the Polish language, which has the best average performance and obtains the best results for three out of nine tasks. Finally, we provide an extensive evaluation, including several standard baselines and recently proposed, multilingual Transformer-based models.
\end{abstract}

\section{Introduction}

The field of natural language understanding (NLU) experienced a major shift towards knowledge reusability and transfer learning, a phenomenon well established in the field of computer vision. Such a shift was enabled by recent introduction of robust, general-purpose models suitable for fine-tuning, like ELMo (Peters et al., 2018), ULMFiT (Howard and Ruder, 2018) and BERT (Devlin et al., 2019).
These models significantly improved the state-ofthe-art on numerous language understanding tasks. Since then, the progress accelerated significantly and the new Transformer-based (Vaswani et al., 2017) models are being published every month to claim the latest state-of-the-art performance.

Such a pace of development would not be possible without standardized and publicly available NLU evaluation benchmarks. Among the most popular ones is the recently introduced GLUE (Wang et al., 2019a) consisting of a collection of tasks such as question answering, sentiment analysis, and textual entailment with texts coming from a diverse set of domains. Some tasks come with numerous training examples, while others have limited training data. On top of that, for some tasks, the training set represents a different domain than the test set. This promotes models that learn general language representations and are effective at transferring knowledge across various tasks and domains. The GLUE benchmark is constructed based on existing datasets, and its main contribution is the careful choice of tasks together with an online evaluation platform and a leaderboard.

Unfortunately, most of the progress in NLU is happening for English and Chinese. Other languages lack both pretrained models and evaluation benchmarks. In this paper, we introduce the comprehensive multi-task benchmark for the Polish language understanding - KLEJ (eng. GLUE, also abbreviation for Kompleksowa Lista Ewaluacji Jezykowych, eng. Comprehensive List of Language Evaluations). KLEJ consists of nine tasks and, similarly to GLUE, is constructed mostly out of existing datasets. The tasks were carefully selected to cover a wide range of genres and different aspects of language understanding. Following GLUE, to 
simplify a model evaluation procedure, we adjusted the tasks to fit into a unified scoring scheme (either text classification or regression). Alongside the benchmark, we introduce HerBERT, a Transformerbased model trained on several Polish text corpora. We compare HerBERT with a set of both standard and recently introduced NLU baselines.

To summarize, our contributions are:

1. KLEJ: A set of nine tasks constructed from both existing and newly introduced datasets used for the Polish language understanding evaluation,

2. An online platform ${ }^{1}$ to evaluate and present the model results in the form of a leaderboard,

3. HerBERT: Transformer-based model for the Polish language understanding,

4. Allegro Reviews: A new sentiment analysis task for the e-commerce domain,

5. Evaluation of several LSTM-based baselines, multilingual Transformer-based models and HerBERT.

The rest of the paper is organized as follows. In Section 2, we provide an overview of related work. In Section 3, we describe the tasks that make up the KLEJ benchmark. In Section 4, we give an overview of the selected baseline methods and introduce the new Transformer-based model for Polish. In Section 5, we evaluate all models using KLEJ benchmark. Finally, we conclude our work in Section 6.

\section{Related Work}

The evaluation of NLU models was always an integral part of their development. Even though there are many established tasks on which to evaluate newly proposed models, there is no strict standard specifying which one to choose. The difficulty of a fair comparison between models eventually led to the introduction of multi-task benchmarks that unify the evaluation.

One such benchmark is SentEval (Conneau and Kiela, 2018). It consists of seventeen established tasks used to evaluate the quality of sentence embeddings. Additionally, ten probing tasks are provided to detect what linguistic properties are retained in sentence embeddings. In all tasks, models

\footnotetext{
${ }^{1}$ https://klejbenchmark.com
}

take either a single sentence embedding or a pair of sentence embeddings as the input and solve a classification (or a regression) problem. The authors released a toolkit ${ }^{2}$ for model evaluation. However, they do not provide a public leaderboard to compare the results of different models.

Another benchmark for evaluating models is decaNLP (McCann et al., 2018), which consists of ten pre-existing tasks. In contrast to SentEval, choice of tasks is much more diverse, ranging from machine translation, semantic parsing to summarization. All tasks have been automatically converted to a question answering format.

Finally, the GLUE benchmark (Wang et al., 2019a) proposes a set of nine tasks. All of them are constructed from existing, well-established datasets. Authors selected tasks that are more diverse and more difficult than SentEval. Otherwise, the design of the benchmark is similar to SentEval.

The aforementioned benchmarks are limited to the English language. Noteworthy attempts at providing multi-language benchmarks include XNLI dataset (Conneau et al., 2018), with the MNLI (Williams et al., 2018) dataset translated by professional translators into 14 languages. A similar effort is XQuAD (Artetxe et al., 2019) which is a translation of the SQuAD dataset (Rajpurkar et al., 2016) into 10 languages.

None of these efforts includes Polish. Other resources to evaluate the Polish language understanding models are scarce. Recently, KrasnowskaKieraś and Wróblewska (2019) prepared their version of the SentEval probing tasks for the Polish language. However, it is more suited for analyzing the sentence embeddings linguistic properties than assessing their quality.

The PolEval (Wawer and Ogrodniczuk, 2017; Kobyliński and Ogrodniczuk, 2017; Ogrodniczuk and Kobyliński, 2018, 2019) ${ }^{3}$ platform organizes an annual competition in natural language processing for the Polish language. During the first three editions, it assembled 11 diverse tasks and attracted over 40 teams. It could serve as the natural benchmark for the Polish language understanding, but it lacks the common interface for all tasks, making it difficult and time-consuming to use. We include one of the PolEval tasks into the KLEJ Benchmark.

Recently Dadas et al. (2019) introduced a benchmark similar to the KLEJ benchmark proposed in

\footnotetext{
${ }^{2}$ https://github.com/facebookresearch/ SentEval

${ }^{3}$ http: //poleval.pl
} 


\begin{tabular}{lrrrlll}
\hline Name & Train & Dev & Test & Domain & Metrics & Objective \\
\hline NKJP-NER & $16 \mathrm{k}$ & $2 \mathrm{k}$ & $2 \mathrm{k}$ & Balanced corpus & Accuracy & NER classification \\
CDSC-R & $8 \mathrm{k}$ & $1 \mathrm{k}$ & $1 \mathrm{k}$ & Image captions & Spearman corr. & Semantic relatedness \\
CDSC-E & $8 \mathrm{k}$ & $1 \mathrm{k}$ & $1 \mathrm{k}$ & Image captions & Accuracy & Textual entailment \\
\hline \multicolumn{6}{c}{ Multi-Sentence Tasks } \\
\hline CBD & $10 \mathrm{k}$ & - & $1 \mathrm{k}$ & Social Media & F1-Score & Cyberbullying detection \\
PolEmo2.0-IN & $6 \mathrm{k}$ & $0.7 \mathrm{k}$ & $0.7 \mathrm{k}$ & Online reviews & Accuracy & Sentiment analysis \\
PolEmo2.0-OUT & $6 \mathrm{k}$ & $0.5 \mathrm{k}$ & $0.5 \mathrm{k}$ & Online reviews & Accuracy & Sentiment analysis \\
Czy wiesz? & $5 \mathrm{k}$ & - & $1 \mathrm{k}$ & Wikipedia & F1-Score & Question answering \\
PSC & $4 \mathrm{k}$ & - & $1 \mathrm{k}$ & News articles & F1-Score & Paraphrase \\
AR & $10 \mathrm{k}$ & $1 \mathrm{k}$ & $1 \mathrm{k}$ & Online reviews & 1 - wMAE & Sentiment analysis \\
\hline
\end{tabular}

Table 1: The overview of tasks in the KLEJ benchmark. It consists almost exclusively of classification tasks, except for CDSC-R and AR which are regression.

this paper. It contains two sentiment analysis tasks, topic classification and a Polish translation of the SICK dataset (Marelli et al., 2014). Similarly to their work, we use the same sentiment analysis dataset, but transform it into a more difficult task. We also use the analogous dataset to SICK but created from scratch for the Polish language. Finally, we considered the topic classification task to be too easy to include into the proposed benchmark. Overall, KLEJ benchmark consists of nine tasks. They are more diverse, cover a wider range of objectives and evaluate not only single sentences but also whole paragraphs.

\section{Tasks}

KLEJ consists of nine Polish language understanding tasks. Similarly to GLUE, we choose tasks from different domains and with different objectives. In contrast to previous benchmarks, we include several tasks that take multiple sentences as input. We decided to focus on tasks which have relatively small datasets - most of them have less than 10k training examples. Moreover, some tasks require extensive external knowledge to solve them. Such a setup promotes knowledge transfer techniques like transfer learning, instead of training separate models for each task from scratch. In effect, KLEJ supports the goal of creating a general model for the Polish language understanding. We present all tasks in the following sections and summarize them in Table 1.

\subsection{NKJP-NER}

We use the human-annotated part of the NKJP (Narodowy Korpus Jezyka Polskiego, eng. National Corpus of Polish) (Przepiórkowski, 2012) to create the named entity classification task.

The original dataset consists of $85 \mathrm{k}$ sentences, randomly selected from a much larger, balanced and representative corpus of contemporary Polish. We use existing human-annotations of named entities to convert the dataset into a named entity classification task. First, we filter out all sentences with entities of more than one type. Then, we randomly assigned sentences into training, development and test sets in such a way, that each named entity appears only in one of the three splits. We decided to split the sentences based on named entities to make the task more difficult. To increase class balance, we undersample the persName class and merge date and time classes. Finally, we sample sentences without any named entity to represent the noEntity class.

The final dataset consists of $20 \mathrm{k}$ sentences and six classes. The task is to predict the presence and type of a named entity. Although the named entity classification task differs from traditional NER task, it has a comparable difficulty and evaluates similar aspects of language understanding. At the same time, it follows the common technical interface as other KLEJ tasks, which makes it easy to use. We use accuracy for evaluation. 


\subsection{CDSC}

The Compositional Distributional Semantics Corpus (Wróblewska and Krasnowska-Kieraś, 2017) consists of pairs of sentences which are humanannotated for semantic relatedness and entailment. Although the main design of the dataset is inspired by SICK, it differs in details. As in SICK, the sentences come from image captions, but the set of chosen images is much more diverse as they come from 46 thematic groups. We prepared two KLEJ tasks based on the CDS Corpus.

\subsubsection{CDSC-R}

The first task is to predict relatedness between a pair of sentences, ranging from 0 (not related) to 5 (very related). The score is the average of scores assigned by three human annotators. We use the Spearman correlation to measure the performance of the model.

\subsubsection{CDSC-E}

The second task uses the textual entailment annotations to predict if the premise entails the hypothesis (entailment), negates the hypothesis (contradiction), or is unrelated (neutral). Even though there is an imbalanced label distribution (most of them are neutral) we follow Krasnowska-Kieraś and Wróblewska (2019) and use accuracy as an evaluation metric.

\subsection{CBD}

The Cyberbullying Detection task (Ptaszynski et al., 2019) was a part of the 2019 edition of the PolEval competition ${ }^{4}$. The goal is to predict whether a given Twitter message is a case of cyberbullying. We use the dataset as-is and use F1-Score to measure the performance of a given model, following the original design of the task.

\subsection{PolEmo2.0}

The PolEmo2.0 (Kocoń et al., 2019) is a dataset of online consumer reviews from four different domains, namely: medicine, hotels, products and university. It is human-annotated on a level of full reviews, as well as individual sentences. It consists of over 8000 reviews, about $85 \%$ of which are from the medicine and hotel domains. Each review is annotated with one of four labels: positive, negative, neutral or ambiguous. The task is to predict the correct label.

\footnotetext{
${ }^{4}$ http://2019.poleval.pl/index.php/ tasks/task 6
}

We use the PolEmo2.0 dataset to form two tasks. Both of them use the same training dataset, i.e. reviews from medicine and hotel domains, but are evaluated on a different test set.

\subsubsection{In-Domain}

In the first task, we use accuracy to evaluate model performance within the in-domain context, i.e. on a test set of reviews from medicine and hotels domains.

\subsubsection{Out-of-Domain}

In the second task, we test the model on out-ofdomain reviews, i.e. from product and university domains. Since the original test sets for those domains are scarce (50 reviews each) we decided to use the original out-of-domain training set of 900 reviews for testing purposes and create the new split of development and test sets. As a result, the task consists of 1000 reviews, which is comparable in size to the in-domain test dataset of 1400 reviews.

\subsection{Czy wiesz?}

The Czy wiesz? (eng. Did you know?) dataset (Marcinczuk et al., 2013) consists of almost 5k question-answer pairs obtained from $C z y$ wiesz... section of Polish Wikipedia. Each question is written by a Wikipedia collaborator and is answered with a link to a relevant Wikipedia article.

The authors of the dataset used it to build a Question Answering system. Then, they evaluated the system using $1.5 \mathrm{k}$ questions. For each question, they took the top 10 system responses and manually annotated if the answer was correct. Positive responses to 250 questions were further processed and only relevant continuous parts of responses were selected by human annotators. Following this procedure, we have manually extracted shorter responses from the remaining positive examples. Finally, we used these annotations to create positive question-answer pairs.

To select the most difficult negative answers, we used the byte-pair encoding (BPE) (Rico Sennrich and Birch., 2016) token overlap between a question and a possible answer. For each question, we took only four most similar negatives and removed ones with a similarity metric score below the threshold $\tau=0.3$. On average, the negative answers were much longer than the positive ones. Since it could be potentially exploited by the model, we decided to balance the length of the positive and negative 
answers. To sample the most relevant part of a negative example, we used BPE based metric with an additional penalty for the number of sentences:

$$
\widehat{\operatorname{sim}}_{\mathrm{BPE}}=\frac{\operatorname{sim}_{\mathrm{BPE}}}{1.2^{\# \text { sents }}}
$$

The task is to predict if the answer to the given question is correct or not. Since the dataset is highly imbalanced, we chose F1-score metric.

\subsection{PSC}

The Polish Summaries Corpus (PSC) (Ogrodniczuk and Kopeć, 2014) is a dataset of summaries for 569 news articles. For each article, the human annotators created five extractive summaries by choosing approximately $5 \%$ of the original text. Each summary was created by a different annotator. The subset of 154 articles was also supplemented with additional five abstractive summaries each, i.e. not created from the fragments of the original article.

Based on PSC we formulate a text-similarity task. We generate the positive pairs (i.e. referring to the same article) using only those news articles which have both extractive and abstractive summaries. We match each extractive summary with two least similar abstractive ones of the same article. We use the same similarity metric as in the preparation of the Czy wiesz? dataset, calculating the BPE token overlap between the extractive and abstractive summary.

To create negative pairs, we follow a similar procedure. For each extractive summary, we find two most similar abstractive summaries, but from different articles. We remove examples with similarity below the threshold $\tau=0.15$. To increase the difficulty and diversity of the task, we filter out multiple abstracts from the same article. As a result, there is at most one negative pair created from each pair of articles.

In total, we obtain around $4 \mathrm{k}$ examples. We randomly split the dataset into train and test based on the articles of the extracts to further increase the task's difficulty. For evaluation, we use F1-score.

\subsection{AR}

We introduce a new sentiment analysis dataset, named Allegro Reviews (AR), extracting $12 \mathrm{k}$ product reviews from Allegro.pl - a popular ecommerce marketplace. Each review is at least 50 words long and has a rating on a scale from one (negative review) to five (positive review). The task is to predict the rating of a given review.

To counter slight class imbalance in the dataset, we propose to evaluate models using $w M A E$, i.e. macro-average of the mean absolute error per class. Additionally, we transform the rating to be between zero and one and report $1-w M A E$ to ensure consistent metric interpretation between tasks.

\section{Baselines}

In this section, we present an overview of several baseline models, which we evaluated using the KLEJ benchmark. We divide these models into three main groups: (1) the LSTM-based (Hochreiter and Schmidhuber, 1997) models using pretrained word embeddings, (2) models based on Transformer architecture and (3) BERT model trained on Polish corpora. We also include the simple baseline by sampling targets from a training set.

\subsection{LSTM-based models}

We chose the standard Bidirectional LSTM text encoder as the base architecture. Following the GLUE experiments setup (Wang et al., 2019a) we trained it jointly as the multi-task learner on all KLEJ tasks.

The architecture consists of two parts: a shared sentence encoder and a task specific classifier. The sentence representation model is a two layer BiLSTM with 1024 hidden units, 300 dimensional word embeddings and max pooling. The classifier is an MLP with 512 dimensional hidden layer.

We perform training in two stages. First, we pretrain the whole model in a multi-task scheme. In the second stage, we freeze the sentence encoder and fine-tune the classifiers separately for each task. The initial learning rate in both phases was set to $10^{-4}$ with linear decay down to $10^{-5}$. Pretraining progress is measured by the macro average of all task metrics. We train models with a batch size of 128, except for the ELMo version, which is trained with a batch size of 64 . For tasks without development set, we use $10 \%$ of training examples as validation data.

We used jiant (Wang et al., 2019b) library to train the LSTM-based models and report the median performance of 5 runs.

\subsubsection{Vanilla BiLSTM}

The simplest version of the LSTM-based models is a BiLSTM sentence encoder with an MLP classifier 
trained from scratch without any form of transfer learning, i.e. without the usage of pretrained word embeddings.

\subsection{2 fast Text}

Before contextual word embeddings became widely adopted, models were enhanced with pretrained word vectors. To evaluate their impact on KLEJ tasks, we initialize word embeddings with fastText (Bojanowski et al., 2016) trained on Common Crawl and Wikipedia for Polish language (Grave et al., 2018).

\subsubsection{ELMo}

ELMo (Peters et al., 2018) is a bidirectional language model using character-level convolutions. In contrast to fastText, ELMo's embeddings capture word-level semantics in a context of the whole sentence.

We conducted more thorough experiments with ELMo embeddings. During the fine-tuning stage in training on a downstream KLEJ task, we modified the sentence encoder parameters and trained the entire architecture with only a word embedding's weights unmodified. Additionally, we experimented with the attention mechanism (Conneau et al., 2017) between all words in tasks with a pair of sentences.

We use publicly available pretrained ELMo weights for Polish language (Janz, 2019).

\subsection{Transformer-based models}

Recently, the best results on the GLUE benchmark were obtained by Transformer-based models inspired by the Bidirectional Encoder Representations (BERT) model. All of them are pretrained on large text corpora using some variant of Masked Language Model (MLM) objective. In this section, we describe three such models: Multilingual BERT, XLM (Lample and Conneau, 2019) and Slavic-BERT (Arkhipov et al., 2019). At the time of writing this paper, these are the only available Transformer-based models that were trained with Polish text.

To evaluate these models we fine-tune them on each task separately. For training we used the transformers (Wolf et al., 2019) library. All models were trained for 4 epochs with a batch size of 32 and using a linearly decaying learning rate scheme starting at $2 \times 10^{-5}$ with a 100 iteration warm-up. We use Adam optimizer with parameters: $\beta_{1}=0.9, \beta_{2}=0.999, \epsilon=10^{-8}$. We report the median performance of 5 runs.

\subsubsection{Multilingual BERT}

The BERT is a popular model based on the Transformer architecture trained using MLM and Next Sentence Prediction (NSP) objectives. We use the Multilingual Cased BERT model, which was trained on 104 languages (including Polish), selecting ones with the largest among all Wikipedia corpora. It uses the shared WordPiece (Wu et al., 2016) tokenizer with the vocabulary size of $110 \mathrm{k}$.

\subsubsection{XLM}

The Cross-lingual Language Model (XLM) is based on BERT. It differs from BERT in that it does not use NSP objective, has more layers (16 vs 12 ), more attention heads (16 vs 12 ), larger hidden layers size (1280 vs 768) and a larger vocabulary (200k vs $110 \mathrm{k}$ ). Moreover, the vocabulary is learned on a corpus for which the most popular languages were undersampled to balance the number of tokens between high- and low-resource languages. We use the XLM-17 model, which was trained on Wikipedia for 17 languages (including Polish).

\subsubsection{Slavic-BERT}

The Slavic-BERT is a BERT model trained on four Slavic languages (Polish, Czech, Russian, and Bulgarian). Contrary to previous models, Arkhipov et al. (2019) used not only Wikipedia but also the Russian News corpus. To avoid costly pretraining, the model was initialized with Multilingual BERT.

\subsection{HerBERT}

None of the above models was optimized for Polish and all of them were trained on Wikipedia only. We decided to combine several publicly available corpora and use them to train a Transformer-based model specifically for the Polish language.

\subsubsection{Corpora}

In this section, we describe the corpora on which we trained our model. Due to copyright constraints we were not able to use the National Corpus of Polish (NKJP), the most commonly known Polish corpus. Instead, we combined several other publicly available corpora and created a larger, but less representative corpus. 


\begin{tabular}{lrrr}
\hline Corpus & Tokens & Texts & Avg len \\
\hline NKJP & $1357 \mathrm{M}$ & $3.9 \mathrm{M}$ & 348 \\
\hline \hline OSCAR & $6710 \mathrm{M}$ & $145 \mathrm{M}$ & 46 \\
Open Subtitles & $1084 \mathrm{M}$ & $1.1 \mathrm{M}$ & 985 \\
Wikipedia & $260 \mathrm{M}$ & $1.5 \mathrm{M}$ & 190 \\
Wolne Lektury & $41 \mathrm{M}$ & $5.5 \mathrm{k}$ & 7450 \\
Allegro Articles & $18 \mathrm{M}$ & $33 \mathrm{k}$ & 552 \\
\hline Total & $8113 \mathrm{M}$ & $150 \mathrm{M}$ & 54 \\
\hline
\end{tabular}

Table 2: Overview of corpora used to train HerBERT compared to the NKJP. Avg len is the average number of tokens per document in each corpus.

Wikipedia Polish version is among the top 10 largest Wikipedia versions. However, it is still relatively small compared to the English one (260M vs $3700 \mathrm{M}$ words). To extract a clean corpus from the raw Wikipedia dump, we used the tools provided by XLM. ${ }^{5}$ However, we did not lowercase the text and did not remove diacritics.

Wolne Lektury (eng. Free Readings) is an online repository of over 5k books, written by Polish authors or translated into Polish. Although the majority of the books in the dataset were written in the 19th or 20th century and they might not be fully representative of the contemporary Polish, they are free to download and can be used as a text corpus.

Open Subtitles is a multilingual parallel corpus based on movie and TV subtitles (Lison and Tiedemann, 2016) from the opensubtitles.org website. As a result, it contains very specific, mostly conversational text consisting of short sentences. Since the translations are communitysourced, they may be of substandard quality. The Polish part of the dataset is relatively large compared to the other corpora (see Table 2).

OSCAR is a multilingual corpus created by Ortiz Suárez et al. (2019) based on Common Crawl ${ }^{6}$. The original dataset lacks information about the language used in particular documents. Categorization to specific languages was automated by a classifier, splitting whole Common Crawl into many monolingual corpora. Duplicates were removed from the dataset to increase its quality. We

\footnotetext{
${ }^{5}$ https://github.com/facebookresearch/ XLM

${ }^{6}$ http: //commoncrawl.org/
}

only use the Polish part of the corpus and use texts longer than 100 words.

Allegro Articles Additionally, we obtained over 30k articles from Allegro.pl - a popular ecommerce marketplace. They contain product reviews, shopping guides and other texts from the ecommerce domain. It is the smallest corpus we've used, but it contains high-quality documents from the domain of our interest.

\subsubsection{Model}

Architecture HerBERT is a multi-layer bidirectional Transformer. We use BERT $\mathrm{BASE}_{\mathrm{BS}}$ architecture configuration with 12 layers, 12 attention heads and hidden dimension of 768 .

Loss We train HerBERT with a MLM objective. According to the updated version of BERT, we always mask all tokens corresponding to the randomly picked word. Whole word masking objective is more difficult to learn than predicting subword tokens (Joshi et al., 2019; Martin et al., 2019).

In the original BERT training setup tokens are masked statically during the text preprocessing phase. In HerBERT, we chose to use dynamic token masking, which follows the training setup of the RoBERTa model (Liu et al., 2019).

We decided not to use the NSP objective. Previous studies by Yang et al. (2019) and Liu et al. (2019) showed that this objective is too easy and does not improve performance on downstream tasks.

Data preprocessing We tokenize corpus data into subword tokens using BPE. We learn BPE splits on Wolne Lektury and a publicly available subset of National Corpus of Polish. We choose these two datasets because of their higher quality compared to the rest of our corpus. We limit the vocabulary size to $50 \mathrm{k}$ tokens.

Our datasets contain a lot of small fragments of coherent text that should be treated as separate documents. We remove degenerated documents that consist of less than 20 tokens from available corpora. Maximal segment length is 512 as it was originally proposed in BERT. We do not accumulate short examples into full 512 token segments because such sequences would be incoherent with frequent topic changes. The only exception to this rule is the Open Subtitles dataset, where subsequent parts of dialogues were connected to form larger documents. The aforementioned training 


\begin{tabular}{|c|c|c|c|c|c|c|c|c|c|c|}
\hline Model & $\stackrel{b}{2}$ & 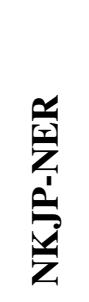 & 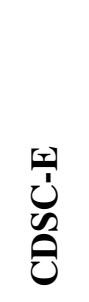 & 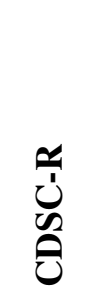 & قิ & 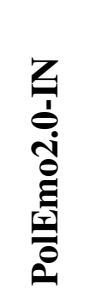 & 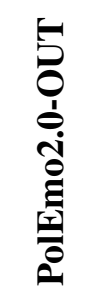 & 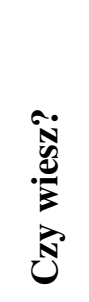 & U & $\frac{\xi}{4}$ \\
\hline Random & 28.3 & 20.7 & 59.2 & 0.9 & 11.2 & 27.8 & 28.5 & 18.9 & 30.4 & 56.9 \\
\hline LSTM & 63.0 & 45.0 & 87.5 & 84.7 & 20.7 & 79.6 & 60.7 & 22.3 & 84.4 & 81.8 \\
\hline LSTM + fastText & 67.7 & 67.3 & 87.8 & 81.6 & 32.8 & 83.2 & 61.1 & 27.5 & 86.5 & 81.4 \\
\hline LSTM + ELMo & 76.6 & 93.0 & 88.9 & 90.4 & 50.2 & 88.5 & 72.1 & 28.8 & 92.7 & 85.1 \\
\hline LSTM + ELMo + fine-tune & 76.7 & 93.4 & 89.3 & 91.1 & 47.9 & 87.4 & 70.6 & 30.9 & 93.7 & 86.2 \\
\hline LSTM + ELMo + attention & 75.8 & 93.0 & 90.0 & 90.3 & 46.8 & 88.8 & 70.2 & 26.0 & 92.1 & 85.4 \\
\hline Multi-BERT & 79.5 & 91.4 & 93.8 & 92.9 & 40.0 & 85.0 & 66.6 & 64.2 & 97.9 & 83.3 \\
\hline Slavic-BERT & 79.8 & 93.3 & 93.7 & 93.3 & 43.1 & 87.1 & 67.6 & 57.4 & 98.3 & 84.3 \\
\hline XLM-17 & 80.2 & 91.9 & 93.7 & 92.0 & 44.8 & 86.3 & 70.6 & 61.8 & 96.3 & 84.5 \\
\hline HerBERT & 80.5 & 92.7 & 92.5 & 91.9 & 50.3 & 89.2 & 76.3 & 52.1 & 95.3 & 84.5 \\
\hline
\end{tabular}

Table 3: Baseline evaluation on KLEJ benchmark. $A V G$ is the average score across all tasks.

setup gives us a slightly better performance on downstream tasks than simply selecting all available data.

Hyperparameters We train HerBERT using Adam optimizer (Kingma and Ba, 2014) with parameters: $\beta_{1}=0.9, \beta_{2}=0.999, \epsilon=10^{-8}$. We use learning rate burn-in over the first 500 steps, reaching a peak value of $10^{-4}$; the learning rate is then linearly decayed for the rest of the training. We train the model with a batch size of 570 . HerBERT was trained for 180k steps, without showing signs of saturation.

\section{Evaluation}

We first compare models based on their average performance. Even though it is not the definite metric to compare models, especially as not all tasks are equally difficult, it gives a general notion of the model performance across all tasks.

In comparison to baselines based on the LSTM architecture, Transformer-based models clearly show superior performance. The only exception is ELMo, which achieves competitive results on many tasks. On two of them, the fine-tuned ELMo model achieves the best score. In general, the evaluation shows major shortcomings of multilingual pretrained BERT models for Polish, and possibly other low resource languages. Overall, every LSTM- based baseline is still on average worse than any of the tested Transformer-based models.

The KLEJ benchmark was designed to require additional knowledge to promote general language understanding models. As expected, we observe significant increases of models quality when using pretrained word embeddings. The vanilla LSTM model achieves the average score of 63.0 while supplying it with the fastText embeddings boosts performance to 67.7. Usage of more recent, contextualized embeddings (ELMo) increases the score to 76.6 .

Focusing on fewer languages seems to result in a better model. The Slavic-BERT has higher scores than Multi-BERT on seven out of nine tasks. However, without a detailed ablation study, it is difficult to infer the main reason resulting in better performance. It can also be attributed to a better tokenizer, additional Russian News corpus or longer training (the Slavic-BERT was initialized with Multi-BERT weights).

The training corpus seems to play an important role in the performance of a downstream task. Both HerBERT and ELMo models were trained mainly on web crawled texts and they excel at tasks from an online domain (CBD, PolEmo-IN, PolEmo-OUT, and $A R$ ). On the other hand, the other Transformerbased models are superior on the Czy wiesz? task. It can be related to the fact that it is a Wikipedia- 
based question-answering task and the aforementioned models were trained mainly on Wikipedia corpus. Interestingly, the Slavic-BERT, which was additionally trained on Russian News corpus, has a lower score on the $C z y$ wiesz? task than MultiBERT and XLM-17.

HerBERT achieves highly competitive results compared to the other Transformer-based models. It has the best performance on average and achieves state-of-the-art results on three tasks, PolEmo-IN, PolEmo-OUT and CBD. Moreover, HerBERT has the smallest performance gap between PolEmo-IN and PolEmo-OUT, which suggests better generalization across domains. Compared to the other Transformer-based models it performs poorly on Czy wiesz? and PSC tasks.

The KLEJ benchmark proved to be challenging and diverse. There is no clear winner among evaluated models; different models perform better at different tasks. It suggests that the KLEJ benchmark is far from being solved, and it can be used to evaluate and compare future models.

\section{Conclusion}

We introduce the KLEJ benchmark, a comprehensive set of evaluation tasks for the Polish language understanding. Its goal is to drive the development of better NLU models, so careful selection of tasks was crucial. We mainly focused on a variety of text genres, objectives, text lengths, and difficulties, which allows us to assess the models across different axes. As a result, KLEJ benchmark proves to be both challenging and diverse, as there is no single model that outperforms others on all tasks.

We find it equally important to provide a common evaluation interface for all the tasks. For that purpose, many existing resources had to be adapted, either automatically (NKJP-NER, PSC) or manually (Czy wiesz?), to make it easier to use.

It's worth mentioning that the main weakness of creating such benchmarks is focusing only on the model performance and not the model efficiency, e.g. in terms of training data, speed or a number of parameters. It seems reasonable to derive additional benchmarks by requiring a given level of efficiency from participating models. We leave it as future work.

We also present HerBERT, a Transformer-based model trained specifically for Polish and compare it with other LSTM- and Transformer-based models. We find that it is the best on average and achieves highest scores on three tasks. We plan to continue the work on HerBERT and use the KLEJ benchmark to guide its development.

\section{References}

Mikhail Arkhipov, Maria Trofimova, Yuri Kuratov, and Alexey Sorokin. 2019. Tuning multilingual transformers for language-specific named entity recognition. In Proceedings of the 7th Workshop on BaltoSlavic Natural Language Processing, pages 89-93, Florence, Italy. Association for Computational Linguistics.

Mikel Artetxe, Sebastian Ruder, and Dani Yogatama. 2019. On the cross-lingual transferability of monolingual representations.

Piotr Bojanowski, Edouard Grave, Armand Joulin, and Tomas Mikolov. 2016. Enriching word vectors with subword information. arXiv preprint arXiv:1607.04606.

Alexis Conneau and Douwe Kiela. 2018. Senteval: An evaluation toolkit for universal sentence representations. In Proceedings of the Eleventh International Conference on Language Resources and Evaluation (LREC-2018).

Alexis Conneau, Douwe Kiela, Holger Schwenk, Loïc Barrault, and Antoine Bordes. 2017. Supervised learning of universal sentence representations from natural language inference data. In Proceedings of the 2017 Conference on Empirical Methods in Natural Language Processing, pages 670-680, Copenhagen, Denmark. Association for Computational Linguistics.

Alexis Conneau, Ruty Rinott, Guillaume Lample, Adina Williams, Samuel R. Bowman, Holger Schwenk, and Veselin Stoyanov. 2018. Xnli: Evaluating crosslingual sentence representations. In Proceedings of the 2018 Conference on Empirical Methods in Natural Language Processing. Association for Computational Linguistics.

Sławomir Dadas, Michał Perełkiewicz, and Rafał Poświata. 2019. Evaluation of sentence representations in polish.

Jacob Devlin, Ming-Wei Chang, Kenton Lee, and Kristina Toutanova. 2019. Bert: Pre-training of deep bidirectional transformers for language understanding. In Proceedings of the 2019 Conference of the North American Chapter of the Association for Computational Linguistics: Human Language Technologies, Volume 1 (Long and Short Papers), pages 4171-4186.

Edouard Grave, Piotr Bojanowski, Prakhar Gupta, Armand Joulin, and Tomas Mikolov. 2018. Learning word vectors for 157 languages. In Proceedings of the International Conference on Language Resources and Evaluation (LREC 2018). 
S. Hochreiter and J. Schmidhuber. 1997. Long shortterm memory. Neural Computation, 9(8):17351780 .

Jeremy Howard and Sebastian Ruder. 2018. Universal language model fine-tuning for text classification. In Proceedings of the 56th Annual Meeting of the Association for Computational Linguistics (Volume 1: Long Papers), pages 328-339.

Arkadiusz Janz. 2019. ELMo embeddings for polish. CLARIN-PL digital repository.

Mandar Joshi, Danqi Chen, Yinhan Liu, Daniel S. Weld, Luke Zettlemoyer, and Omer Levy. 2019. Spanbert: Improving pre-training by representing and predicting spans. arXiv preprint arXiv:1907.10529.

Diederik P. Kingma and Jimmy Ba. 2014. Adam: A method for stochastic optimization. Cite arxiv:1412.6980Comment: Published as a conference paper at the 3rd International Conference for Learning Representations, San Diego, 2015.

Łukasz Kobyliński and Maciej Ogrodniczuk. 2017. Results of the poleval 2017 competition: Part-ofspeech tagging shared task. In Proceedings of the 8th Language and Technology Conference: Human Language Technologies as a Challenge for Computer Science and Linguistics, pages 362-366.

Jan Kocoń, Piotr Miłkowski, and Monika ZaśkoZielińska. 2019. Multi-level sentiment analysis of PolEmo 2.0: Extended corpus of multi-domain consumer reviews. In Proceedings of the 23rd Conference on Computational Natural Language Learning (CoNLL), pages 980-991, Hong Kong, China. Association for Computational Linguistics.

Katarzyna Krasnowska-Kieraś and Alina Wróblewska. 2019. Empirical linguistic study of sentence embeddings. In Proceedings of the 57th Annual Meeting of the Association for Computational Linguistics, pages 5729-5739.

Guillaume Lample and Alexis Conneau. 2019. Crosslingual language model pretraining. arXiv preprint arXiv:1901.07291.

Pierre Lison and Jörg Tiedemann. 2016. Opensubtitles2016: Extracting large parallel corpora from movie and tv subtitles. In Proceedings of the Tenth International Conference on Language Resources and Evaluation (LREC 2016), Paris, France. European Language Resources Association (ELRA).

Yinhan Liu, Myle Ott, Naman Goyal, Jingfei Du, Mandar Joshi, Danqi Chen, Omer Levy, Mike Lewis, Luke Zettlemoyer, and Veselin Stoyanov. 2019. Roberta: A robustly optimized bert pretraining approach. arXiv preprint arXiv:1907.11692.

Michał Marcinczuk, Marcin Ptak, Adam Radziszewski, and Maciej Piasecki. 2013. Open dataset for development of polish question answering systems.
In Proceedings of the 6th Language \& Technology Conference: Human Language Technologies as a Challenge for Computer Science and Linguistics, Wydawnictwo Poznanskie, Fundacja Uniwersytetu im. Adama Mickiewicza.

Marco Marelli, Stefano Menini, Marco Baroni, Luisa Bentivogli, Raffaella Bernardi, and Roberto Zamparelli. 2014. The sick (sentences involving compositional knowledge) dataset for relatedness and entailment.

Louis Martin, Benjamin Muller, Pedro Javier Ortiz Suárez, Yoann Dupont, Laurent Romary, Éric Villemonte de la Clergerie, Djamé Seddah, and Benoît Sagot. 2019. CamemBERT: a Tasty French Language Model. arXiv e-prints, page arXiv:1911.03894.

Bryan McCann, Nitish Shirish Keskar, Caiming Xiong, and Richard Socher. 2018. The natural language decathlon: Multitask learning as question answering. arXiv preprint arXiv:1806.08730.

Maciej Ogrodniczuk and Łukasz Kobyliński, editors. 2018. Proceedings of the PolEval 2018 Workshop. Institute of Computer Science, Polish Academy of Sciences, Warsaw, Poland.

Maciej Ogrodniczuk and Łukasz Kobyliński, editors. 2019. Proceedings of the PolEval 2019 Workshop. Institute of Computer Science, Polish Academy of Sciences, Warsaw, Poland.

Maciej Ogrodniczuk and Mateusz Kopeć. 2014. The Polish Summaries Corpus. In Proceedings of the Ninth International Conference on Language Resources and Evaluation, LREC 2014.

Pedro Javier Ortiz Suárez, Benoît Sagot, and Laurent Romary. 2019. Asynchronous Pipeline for Processing Huge Corpora on Medium to Low Resource Infrastructures. In 7th Workshop on the Challenges in the Management of Large Corpora (CMLC7), Cardiff, United Kingdom. Leibniz-Institut für Deutsche Sprache.

Matthew E. Peters, Mark Neumann, Mohit Iyyer, Matt Gardner, Christopher Clark, Kenton Lee, and Luke Zettlemoyer. 2018. Deep contextualized word representations. In Proc. of NAACL.

Adam Przepiórkowski. 2012. Narodowy korpus jezyka polskiego. Naukowe PWN.

Michal Ptaszynski, Agata Pieciukiewicz, and Paweł Dybała. 2019. Results of the poleval 2019 shared task 6: First dataset and open shared task for automatic cyberbullying detection in polish twitter. Proceedings of the PolEval 2019 Workshop, page 89

Pranav Rajpurkar, Jian Zhang, Konstantin Lopyrev, and Percy Liang. 2016. Squad: 100,000+ questions for machine comprehension of text. In Proceedings of the 2016 Conference on Empirical Methods in Natural Language Processing, pages 2383-2392. 
Barry Haddow Rico Sennrich and Alexandra Birch. 2016. Neural machine translation of rare words with subword units. In Association for Computational Linguistics (ACL), pages 1715-1725.

Ashish Vaswani, Noam Shazeer, Niki Parmar, Jakob Uszkoreit, Llion Jones, Aidan N Gomez, Ł ukasz Kaiser, and Illia Polosukhin. 2017. Attention is all you need. In I. Guyon, U. V. Luxburg, S. Bengio, H. Wallach, R. Fergus, S. Vishwanathan, and R. Garnett, editors, Advances in Neural Information Processing Systems 30, pages 5998-6008. Curran Associates, Inc.

Alex Wang, Amanpreet Singh, Julian Michael, Felix Hill, Omer Levy, and Samuel R. Bowman. 2019a. GLUE: A multi-task benchmark and analysis platform for natural language understanding. In the Proceedings of ICLR.

Alex Wang, Ian F. Tenney, Yada Pruksachatkun, Katherin Yu, Jan Hula, Patrick Xia, Raghu Pappagari, Shuning Jin, R. Thomas McCoy, Roma Patel, Yinghui Huang, Jason Phang, Edouard Grave, Haokun Liu, Najoung Kim, Phu Mon Htut, Thibault F'evry, Berlin Chen, Nikita Nangia, Anhad Mohananey, Katharina Kann, Shikha Bordia, Nicolas Patry, David Benton, Ellie Pavlick, and Samuel R. Bowman. 2019b. jiant 1.2: A software toolkit for research on general-purpose text understanding models. http://jiant.info/.

Aleksander Wawer and Maciej Ogrodniczuk. 2017. Results of the poleval 2017 competition: Sentiment analysis shared task. In 8th Language and Technology Conference: Human Language Technologies as a Challenge for Computer Science and Linguistics.

Adina Williams, Nikita Nangia, and Samuel Bowman. 2018. A broad-coverage challenge corpus for sentence understanding through inference. In Proceedings of the 2018 Conference of the North American Chapter of the Association for Computational Linguistics: Human Language Technologies, Volume 1 (Long Papers), pages 1112-1122. Association for Computational Linguistics.

Thomas Wolf, Lysandre Debut, Victor Sanh, Julien Chaumond, Clement Delangue, Anthony Moi, Pierric Cistac, Tim Rault, R'emi Louf, Morgan Funtowicz, and Jamie Brew. 2019. Huggingface's transformers: State-of-the-art natural language processing. ArXiv, abs/1910.03771.

Alina Wróblewska and Katarzyna Krasnowska-Kieraś. 2017. Polish evaluation dataset for compositional distributional semantics models. In Proceedings of the 55th Annual Meeting of the Association for Computational Linguistics (Volume 1: Long Papers), pages 784-792.

Yonghui Wu, Mike Schuster, Zhifeng Chen, Quoc V Le, Mohammad Norouzi, Wolfgang Macherey, Maxim Krikun, Yuan Cao, Qin Gao, Klaus Macherey, et al. 2016. Google's neural machine translation system: Bridging the gap between human and machine translation. arXiv preprint arXiv:1609.08144.

Zhilin Yang, Zihang Dai, Yiming Yang, Jaime Carbonell, Ruslan Salakhutdinov, and Quoc V Le. 2019. Xlnet: Generalized autoregressive pretraining for language understanding. arXiv preprint arXiv:1906.0823. 